\title{
Studies on Thick Sections of the Nucleus of Mouse Sertoli Cells Using an Electron Microscope Operating at $300 \mathrm{kV}$
}

\author{
By \\ Tsuyuka KUSHIDA, Haruyuki IIJIMA, Yasukazu NAGATO* and Hiroshi KUSHIDA* \\ Department of Anatomy, Tokyo Women's Medical College, Tokyo, 162 Japan \\ *Department of Morphology, Division of Human Structure and Function, School of Medicine, Tokai University, Isehara \\ 259-11, Japan
}

- Received for Publication, February 12, $1993-$

Key Words: Sertoli cell, Nucleolus, Three-dimensional structure, High voltage electron microscopy

\begin{abstract}
Summary: Examination of the three-dimensional structure of the Sertoli cell nucleus from mouse testes was performed under a high voltage electron microscope operating at $300 \mathrm{kV}$. Using an en bloc staining method along with fixation by osmium tetroxide and embedding in a mixture of Quetol 651, NSA and MNA, the structures of the nucleus were stained at a high contrast and satisafactory preservation was achieved, thus allowing their study at a high resolution within thick sections.

Nuclear components could be observed clearly in 2-3 $\mu$ m-thick sections of embedded material. Typical threedimensional configurations of nucleoli and associated bodies were indicated. Thick sections permitted the observation that two or three perinucleolar bodies are usually attached on each side of the nucleoli or form a triangular shape of different sizes of vacuolar structures within the bodies. Stereoscopic observations also revealed overlapping of perinucleolar bodies and nucleoli and suggested the complexity of the components of perinucleolar and intranucleolar chromatin.
\end{abstract}

Since the introduction of electron microscopy, ultrastructural observations of Sertoli cells have revealed several morphological aspects concerning their function in seminiferous tubules, such as spermatogenesis, phagocytosis and mechanical support for the germ cells (Fawcett and Burgos, 1956; Sapsford, 1963; Kushida, 1964; Nagano, 1966; Flickinger, 1967; Cooksey and Rothwell, 1972; Dym, 1973; Schulze, 1974; Nistal et al., 1982; Wong and Russell, 1983; Weber et al., 1983). Using this approach, the architecture of the Sertoli cell nucleus has also been described in mammalian species (Bustos-Obregon and Esponda, 1974; Krimer, 1977; Mirre and Knibiehler 1982; Paniaqua et al., 1986). Therefore, morphological characteristics such as the nucleolus, perinucleolar body, round body, coiled body and mykaryon have been observed at the ultrastructural level (Jean et al., 1983; Schultz et al., 1984; Schultz, 1989). This evidence was mainly based on examination of thin sections that revealed only parts of the Sertoli cell nucleus.

The nucleolar complex is one of the most prominent structures observed in the mouse Sertoli cell nucleus. Perinucleolar bodies (perinucleolar spheres, satellite karyosomes and heteropyknotic bodies) were considered components of the nucleolus and formed of condensed chromatin, suggesting that these structures are formed by the association of centromeric heterochromatin (Jean et al., 1983). Although the perinucleolar bodies consist of a more or less discontinuous layer of clusters of fibrils usually composed of coiled fine filaments, the precise configuration of the nucleus and associated bodies could not, however, be deduced by the thin sectioning method due to their size and complexity.

Electron microscopy of thick sections can provide three-dimensional information concerning the structural arrangement of cell components (Glauert, 1974) since technical difficulties with serial thin sections are inherent in this approach (Rieder, 1981). Examination of three-dimensional structure determines structure, structural relationships, and changes in these parameters in an effort to comprehend function and mechanism of function (Rieder et al., 1985). When viewed with a high voltage electron microscope, however, the overlapping of cell constituents in sections thicker than $0.5-1.0 \mu \mathrm{m}$ often hides or obscures the structure of complicated cell components observed in stereo. All of the details within the thickness of a stained section are brought into focus as a superimposed image. The superimposition of details in the projected images is a serious problem (Rieder et al., 1985).

Recently, it has become possible to overcome this confusion with the development of useful embedding media and staining procedures suitable for obser- 
vation with an accelerating potential of 200,300 and $400 \mathrm{kV}$ (Kushida et al., 1984; 1987; 1988a). Kushida (1988b) reported that embedding media for stereoscopic observation of semithin or thick tissue sections must be stable and have low electron scattering properties under electron beams at different potentials. The embedding method employing Quetol 651 was reported for the examination of thick sections $1-3 \mu \mathrm{m}$ in thickness using $300 \mathrm{kV}$. This method has been improved by the introduction of en bloc staining and stereoscopic observation, and has been used to examine three-dimensional arrangement of midpieces of spermatozoa (Kushida et al., 1989; 1990).

The study described here establishes the threedimensional structure of the Sertoli cell nucleus, showing the relation of the nucleolus, perinucleolar body and other structures.

\section{Materials and Methods}

\section{Tissue preparations}

En bloc staining with uranyl acetate and epoxy resins, which has low electron scattering properties suitable for observations by electron microscopy at $300 \mathrm{kV}$, was used according to the methods outlined in Kushida et al., (1988a, 1991).

Testes from adult male mice $(\mathrm{C} 3 \mathrm{H} / \mathrm{He})$, which were deeply anesthetized with ether, were prepared for electron microscopic observation. The testis was removed, diced with a razor blade into $1.5 \mathrm{~mm}$ cubes, and immediately fixed by immersion in $2 \%$ buffered osmium tetroxide for $3 \mathrm{hr}$ at $4^{\circ} \mathrm{C}$. After washing in 3 changes of distilled water for $1 \mathrm{hr}$, they were transferred to $1 \%$ aqueous carbohydrazide for $2 \mathrm{hr}$ at room temperature. Then the tissue was washed 3 times in distilled water for $1 \mathrm{hr}$.

To enhance the contrast of the materials in thick sections, staining with osmium tetroxide and uranyl acetate was performed using 1) a $1.0 \%$ aqueous solution of osmium tetroxide for $2 \mathrm{hr}$. and 2) a $2.5 \%$ solution of uranyl acetate in $50 \%$ alcohol for $3 \mathrm{hr}$. After this staining procedure, the tissue was dehydrated in a graded alcohol series and embedded in a mixture of epoxy resin, Quetol 651, NSA and MNA (Kushida et al. 1988a). The mixture sets in about $24 \mathrm{hr}$ at $60^{\circ} \mathrm{C}$ and yields a light yellow block. It has low electron scattering properties under electron beams at accelerating potentials of $300 \mathrm{kV}$ with a conventional electron microscope.

Sections $2-3 \mu \mathrm{m}$ in thickness were cut with glass knives on a conventional ultramicrotome, and mounted on chloroprene rubber-coated grids for electron microscopy without double staining.

\section{Electron microscopy}

A Hitachi H-9000 electron microscope with a side-entry goniometer was used for observations at an accelerating potential of $300 \mathrm{kV}$. The sections were examined at a tilt angle of $\pm 10^{\circ}$, and stereoscopic electron micrographs were obtained. Threedimensional organization was observed using Stereoscope W-101.

\section{Results}

Figure 1 shows a $2.0 \mu \mathrm{m}$ thick section of a mouse seminiferous tubule. An elongated nucleus was usually located at the base of the Sertoli cell, close to the basal membrane of the seminiferous epithelium. Under low magnification of an electron microscope operating at $300 \mathrm{kV}$, the nucleus showed a single, large, roughly spherical, centrally located nucleolus, which was flanked by two or three perinucleolar bodies.

The nucleolus stands out very clearly at high density and is formed by two components: dense, thick cords, composed of fibrillar material and granular centers, which are relatively less dense, enclosed by the thick cords (Figs. 2-8).

The nucleolus has one or more perinucleolar bodies regularly attached in opposite positions (Figs. 1-10). In thick sections, nucleolar bodies show several kinds of distribution patterns, which were not observed in ordinary thin sectons. Figures 3 and 4 exhibit two typical perinucleolar bodies on both sides of the nucleolus. Both bodies, at angles from $110^{\circ}$ to $130^{\circ}$, contain large vacuolar spaces showing very low electron density in the central portion. Dense thick cords cover the body and low density vacuolar spaces are found between the dense cords. The size of the vacuolar spaces varied although one or two prominent vacuoles were present. In random sections, the characteristic triangular arrangement is seen (Figs. 4 and 5). With this method, these sections reveal the structural relationships between the nucleolus and overlaying or underlaying perinucleolar bodies. There is a central round nucleolus, flanked by three smaller round bodies with denser cores. Similar dense components are frequently found in the nucleoplasm without any structural relation to the nucleolus (Fig. 7). They are often closely associated with the nuclear membrane (Fig. 2). Occasionally, the nucleus of Sertoli cells had a single perinucleolar body (Figs 6 and 7), and in rare cases, no bodies flanking the nucleoli were observed in the section (Fig. 8).

The three-dimensional organization of the nucleolus and associated bodies is clearly seen in Figures 9 and 10 . The tilting angle was $\pm 10^{\circ}$. Observations 
with a stereoscope indicated that the perinucleolar bodies clearly overlapped the nucleolus. It was evident that the relation of the three bodies with the nucleolus is retained in thick sections after the fixation and embedding procedures.

The Sertoli cell nucleus at high magnification ususally contained fine structures of various densities throughout the nucleoplasm (Figs 2, 4, 6, 7 and 8). There were roughly ovoidal, spherical and slightly elongated structures, which may be called coiled body or mykaryon in thin section. They were not enclosed by a membrane and were formed of distinct fine fibrillar elements which show a moderate affinity for heavy metal salts. It was obvious that many of them were free in the nucleoplasm, but occasionally, some components in close proximity to the nucleolus were in contact with the pars fibrosa or pars granulosa (Fig. 11). Under high magnification they were found to be composed of a three-dimensional network of elements, although the form and distribution was irregular. Some components contained dense granular regions (Fig. 12).

\section{Discussion}

\section{Introduction of thick sections}

Fixation, embedding and staining procedures for electron microscopic studies influence the quality of the resulting images of nucleolar components. Therefore, fixation with aldehydes followed by fixation with osmium tetroxide was considered to be the best choice for the presevation of nucleolar structures in thin sectioning. Development of epoxy embedding and double-staining methods also had favorable effects on electron microscopic images in the field of thin sections. However, such sections provide only adequate two-dimensional structural information on various cellular components. To determine structure and structural relationships, three-dimensional information is required. Since technical difficulties were revealed through reconstructions from serial thin sections (Rieder, 1981), semithin or thick sections have been used in high voltage electron microscopy when specific ultrastructural information is required that can not be obtained by thin sections.

The development of high voltage $(200-400 \mathrm{kV})$ electron microscopy now enables investigators to examine sections which are appreciably thicker than the conventional thin sections rather than using very high voltages $(500-1,000 \mathrm{kV})$. The usefulness of this procedure was established in previous studies (Kushida et al., 1985; Nagato et al., 1990, 1991). More recently, the introduction of several embedding media, such as a water-miscible methacrylate and a low viscosity epoxy resin, provides suitable conditions for examining tissue sections at different accelerating potentials: these include a mixture of 2-hydroxypropyl methacrylate, Quetol 523 and methyl methacrylate (Kushida et al., 1985), and a mixture of Quetol 812 and NSA for $200 \mathrm{kV}$ (Kushida et al., 1984); a mixture of Quetol 651, NSA and MNA for $300 \mathrm{kV}$ (Kushida et al., 1988a); a mixture of Quetol 651, ERL 4206 and NSA for $400 \mathrm{kV}$ (Kushida et al., 1987).

In stereoscopic observations of thick sections, contrast enhancement in the cellular structures is a serious problem. Therefore, en bloc staining and double staining with uranyl and lead were generally used for enhancing the contrast of images within the sections (Glauert, 1974; Rieder et al., 1985). This study demonstrated that contrast of nuclear structures of Sertoli cells was higher and their preservation was satisfactory when specimens were fixed with osmium tetroxide and stained en bloc with osmium tetroxide and uranyl acetate according to the method of Kushida et al. (1988a).

We introduced a new embedding media using Quetol 651 as well as improved fixation and staining procedures to improve the preservation of the threedimensional configuration of the Sertoli cell nucleous. The results indicated the usefulness of studying thick sections to describe the three-dimensional structure of the nucleus which is not possible with thin sections. With this method, three-dimensional data on nucleolar structure were obtained for the first time from thick sections of Sertoli cells.

\section{Structure of Sertoli cell nucleus in thick sections}

Numerous studies have been performed on various aspects of Sertoli cell structure with thin sections. These examinations permitted an analysis of the nucleolar organization and indicated the existence of nucleoli. heterochromatin bodies, nuclear bodies, coiled bodies and mykaryon (Schulze, 1974; Krimer, 1977; Jean et al., 1983; Schultz, 1989). Thus, four principal components were recognized in the Sertoli cells of mice (Paniaqua et al., 1986): 1) a spherical fibrillar center, 2) a compact granular portion. 3) a reticular portion with intermingled pars fibrosa and pars granulosa and 4) a nucleolar associated chromatin body surrounding the nucleolus. The architecture of these components were not, however, well established since many studies relied on thin sections with randomly cut parts of the Sertoli cell nucleus. When the organization of the mouse Sertoli cell nucleus was reexamined under a high voltage electron microscope operating at $300 \mathrm{kV}$, some morphological aspects including three-dimensional configuration of the nucleolar structure, which have not previously been reported, were revealed. 
It is well known from earlier electron microscope that the nucleolar complex is one of the most prominent structure observed in mouse Sertoli cell nuclei (Krimer, 1977). A single central nucleolus known to be rich in RNA, and perinucleolar bodies were considered components of the nucleolus which was described as a tripartite structure (Flickinger, 1967). Observations with deep staining by the Feulgen reaction (Sapsford, 1963) as well as a negative reaction after the uranyl- EDTA-lead technique (Krimer, 1977) strongly suggested that the perinucleolar bodies are formed of condensed chromatin. If was also suggested that centromeric heterochromatin is involved in the formation of these structures, showing hybridization of satellite DNA in the perinucleolar bodies (Krimer, 1977). In a study reported by $\mathrm{Hsu}$ et al. (1971), it was noted that in mouse Sertoli cells, neurons, and spermatids, all paracentromeric heterochromatin aggregated in one area or in a few neighboring areas. They also reverled that an average of four heterochromatin blocks were found in Sertoli cell nucleoli, which is so fewer than that in immature cell types. Terefore, they suggested that there was a tendency for more differentiated cells to possess fewer heterochromatin blocks than cells with a potential to proliferate. Jean et al. (1983) found that a random-bred Swiss OF-1 strain showed dispersion of parts of the centromeric heterochromatin in granules of unequal size throughout the nucleoplasm, although centromeric heterochromatin was condensed in two perinucleolar bodies in most strains of mice.

In the present study two or three perinucleolar bodies composed of fine filamentous structures were mainly observed. Their sizes varied and they sometimes contained vacuolar spaces of different sizes. Such findings agree with the observation of the previous workers (Hsu, 1971; Krimer, 1977) who chiefly used thin sections. Stereoscopic observation of thick sections revealed that two associated bodies were typically located on each side of a single nucleolus with relatively higher density than the nucleoli, while in the case of three bodies they formed triangular shape. Moreover, thick sections permit observation of overlapping of the perinucleolar bodies with the central nucleoli in the peripheral areas. Such direct interconnection of perinucleolar bodies and the nucleolus indicates that perinucleolar chromatin is apparently an integral part of the nucleolus as suggested by thin section observations (Busch, 1974). Busch (1974) also summarized the results by means of classic techniques in electron microscopy and cytochemical procedures: 1) studies on isolated nucleoli as well as on nucleoli in situ showed that the perinucleolar chromatin is connected with the intranucleolar chromatin by septalike structures or strips which penetrate the nucleolar body complexity between the heterochromatin component and the central nucleolus, 2) penetration of fine solitary fibrils of filaments into the nucleolar body from the nucleolus-associated chromatin was noted, and 3) intranucleolar as well as perinucleolar chromatin may be involved in RNA synthesis. The close structural relation between perinucleolar bodies and nucleolus supports the results of the previous investigations. It also suggests that perinucleolar chromatin is associated with the intranucleolar chromatin in the nucleus. The formation of the perinucleolar bodies or heterochromatin is still unclear in the present study.

Schultz (1989) reported a coiled body and mykaryon in the nucleus of the adult rat Sertoli cell using thin sections. He noted that the coiled body was composed of distinct elements referred to as "coils" and resembled the nucleolar pars fibrosa in their intense staining with heavy metal salts. The mykaryon was composed of a three-dimensional network of "cords" separated by slightly thinner spaces. A coiled body associated with nucleoli was also described in facial motor neurons of the golden hamster (Kinderman and LaVelle, 1976).

The present findings indicate that the Sertoli cell nuclei show a characteristic distribution of some dense bodies, which closely resemble the coiled body described by Schultz (1989), but they are occasionally attached to the nucleolus. However, the previous study demonstrated that the coiled body exists in situ without structural relationship to the nucleolus. In a random-bred adult OF-1 mice, Jean et al. (1983) reported that heterochromatin was seen in granules dispersed in the nucleoplasm. Therefore, the coiled body may reflect the appearance of low concentration masses of dispersed heterochromatin in thick sections.

In conclusion, two or three perinucleolar bodies are in regular contact with each nucleolus. Their overlapping with the nucleolus is a normal structural relationship of the nucleus in Sertoli cells of adult mice. It is clear that other dense componets are attached to the nucleoli. Some ovoidal, spherical and elongated structures are also found in the nucleoplasm.

\section{References}

1) Bustos-Obregon, E., Esponda, P.: Ultrastructure of the nucleus of human Sertoli cells in normal and pathological tissues. Cell Tissue Res., 152: 467-475, 1974.

2) Cooksey, E. J., Rothwell, B.: The ultrastructure of the Sertoli cell its differentiation in the domestic fowl. J. Anat., 114: $329-345,1973$.

3) Dym, M.: The fine structure of the monkey (Macaca) Sertoli 
cell and its role in maintaining the blood-testis barrier. Anat. Rec., 175: 639-656, 1973.

4) Fawcett, D. W., Burgos, M. H.: The fine structure of Sertoli cells in the human testis. Anat. Rec., 124: 401-402, 1956.

5) Flickinger, C. J.: The postnatal development of the Sertoli cells of the mouse. Z. Zellforsch., 78: 92-113, 1967.

6) Glauert, A. M.: The high voltage electron microscope in biology. J. Cell Biol., 63: 717-748, 1974.

7) Hsu, T. C., Cooper, J. E. K., Mace, Jr., Brinkeley, B. R.: Arrangement of centromeres in mouse cells. Chromosoma, 34: 73-87, 1971.

8) Jean, P., Hartung, M., Mirre, C., Stahl, A.: Association of centromeric heterochromatin with the nucleolus in mouse Sertoli cells. Anat. Rec., 205: 375-380, 1983.

9) Kinderman, N. B., LaVelle, A.: A nucleolus-associated coiled body. J. Neurocytol., 5: 545-550, 1976.

10) Krimer, D. B.: Ultrastructural and cytochemical aspects of nucleus of mouse Sertoli cells. Arch. Biol., 88: 117-126, 1977.

11) Kushida, T.: Some observations on the structure of Sertoli cell. Acta Anat. Nippon., 39(supple): 11-12, 1964. (in Japanese).

12) Kushida, H., Kushida, T., Iijima, H., Yonehara, K., Nakazawa, E.: Double staining available for stereoscopic observation of epoxy resin Quetol 812-embedded semithin sections under the $200 \mathrm{kV}$ transmission electron microscope. J. Electron Microsc., 33: 191-194, 1984.

13) Kushida, H., Kushida, T., Iijima, H.: An improved method for both light and electron microscopy of identical sites in semithin tissue sections under a $200 \mathrm{kV}$ transmission electron microscope. J. Electron Microsc., 34: 438-441, 1985.

14) Kushida, H., Kushida, T., Iijima, H., Aita, S.: An improved method for embedding with Quetol 651 and ERL 4206 for stereoscopic observation of thick sections under a $400 \mathrm{kV}$ transmission electron microscope. J. Electron Microsc., 36: 133-135, 1987.

15) Kushida, T., Iijima, H., Kushida, H., Tsuruta, C.: An improved embedding method employing epoxy resin Quetol 651 for stereoscopic observation of thick sections under a $300 \mathrm{kV}$ transmission electron. microscope. J. Electron Microsc., 37: 212-214, 1988a.

16) Kushida, H.: Embedding method for high voltage electron microscopy in biology. Denshi Kenbikyo, 23: 169-173. 1988b. (in Japanese)

17) Kushida, T., Iijima, H., Kushida, H., Tsuruta, C.: Stercoscopic observation of thick sections of spermatocytes under a $300 \mathrm{kV}$ transmission electron microscope. J. Electron microsc., 38: 301, 1989.

18) Kushida, T., Iijima, H., Kushida, H.: Threc-dimensional ultrastructure of spermatogenic cells under a $300 \mathrm{kV}$ transmission electron microscope. Acta Anat. Nippon., 65: 279, 1990. (in Japanese).

19) Kushida, T., Iijima, H., Kushida, H., Tsuruta. C.: En bloc staining available for stereoscopic observation of epoxy resin
Quetol 651-embedded thick sections under a high voltage transmission electron microscope. J. Electron Microsc. . 40: 76-77, 1991.

20) Mirre, C., Knibiehler, B.: A re-evaluation of the relationships between the fibrillar centers and the nucleolus-organizing regions in reticulated nucleoli: Ultrastructural organization. number and distribution of the fibrillar centers in the nucleolus of the mouse Sertoli cell. J. Cell Sci.. 55: 247-259. 1982.

21) Nagano, T.: Some observations on the fine structure of the Sertoli cell in the human testis. Z. Zellforsch., 73: 89-106. 1966.

22) Nagato, Y., Shimai, K., Kushida, T., Kushida, H.: Staining of intestinal goblet cells with ruthenium red in semithin sections. J. Electron Microsc., 39: 115-119, 1990.

23) Nagato, Y., Sekiguchi, M., Kushida, T., Kushida, H., Shimai, K.: Correlative light and electron microscopic observations on ectopic neurons in the cerebellum of dreher mutant mouse. J. Electron Microsc., 40: 11-18, 1991.

24) Nistal, M., Abaurrea, M. A., Paniaqua, R.: Morphological and histometric study on the human Sertoli cell from birth to the onset of puberty. J. Anat., 134: 351-363, 1982.

25) Paniagua, R., Nistal, M., Amat, P., Rodriguez, M. C.: Ultrastructural observations on nucleoli and related structures during human spermatogenesis. Anat. Embryol., 174: 301-306, 1986.

26) Rieder, C. L.: Thick and thin serial sectioning for the threedimensional reconstruction of biological ultrastructure. Meth. Cell Biol., 22: 215-249, 1981.

27) Rieder, C. L., Rupp, G., Bowser, S. S.: Electron microscopy of semithick sections: Advantages for biological research. J. Electron Microsc. Tech., 2: 11-28, 1985.

28) Sapsford, C. S.: The development of the Sertoli cell of the rat and mouse; its existence as a mononucleate unit. $J$. Anat., 97: 225-238, 1963.

29) Schultz, M. C., Hermo, L., Leblond, C. P.: Structure. development and cytochemical properties of the nucleolusassociated "round body" in spermatocytes and early spermatids of the rat. Am. J. Anat.. 171: 41-57, 1984.

30) Schultz, M. C.: Ultrastructural study of the coiled body and a new inclusion, the "mykaryon" in the nucleus of the adult rat Sertoli cell. Anat. Rec., 225: 21-25. 1989.

31) Suhulze, C.: On the morphology of the human Sertoli cell Cell Tissue Res., 153: 339-355, 1974.

32) Smetana, K., Busch, H.: The nucleolus and nucleolar DNA In Busch H. (ed), The Cell Nucleus., Vol. I, Academic Press, New York and London, pp73-147, 1974

33) Weber, J. E., Russell, L. D., Wong, V., Reterson. R. N.: Three-dimensional reconstruction of a rat stage V. Sertoli cell: II Morphometry of Sertoli-Sertoli and Sertoli-germ cell relationships. Am. J. Anat.. 167: 163-179, 1983.

34) Wong, V.. Russell, L. D.: Three-dimensional reconstruction of a rat stage V. Sertoli cell: I Methods, basic configuration and dimensions. Am. J. Anat.. 167: 147-161, 1953 


\section{Explanation of Figures}

\section{Plate I}

Fig. 1. Low magnification of a cross section of a seminiferous tubule from adult mouse testis. A $2.0 \mu \mathrm{m}$ section shows Sertoli cell nuclei with their characteristic nucleoli.

Fig. 2. A Sertoli cell nucleus shows typical nucleolus $(\mathrm{Nu})$ with two perinucleolar bodies (PB) and some dense fine components $\left(^{*}\right)$ near the nucleolar complex. A dense body closely associated with the nuclear membrane is seen. $2.0 \mu \mathrm{m}$ section.

Fig. 3. A $2.0 \mu \mathrm{m}$ section shows the nuclear detail with a high magnification. A prominent nucleolar complex is seen, formed by a nucleolus (Nu) and two perinucleolar bodies (PB) on both sides. Both bodies contain vacuolar spaces.

Fig. 4. Electron micrograph showing a nucleolus in close contact with three perinucleolar bodies. The three bodies form triangular shape. A large vacuolar space is found within the body. Dense structures $(*)$ are present near the nucleolar complex. $2.0 \mu \mathrm{m}$ section.

Fig. 5. Overlapping of three perinucleolar bodies with the central nucleolus $(\mathrm{Nu})$ is apparent within the section, $3.0 \mu \mathrm{m}$ in thickness.

Fig. 6. Electron micrograph showing a nucleolus with one associated body. Overlapping of the structures is not clear. $2.0 \mu \mathrm{m}$ section. 

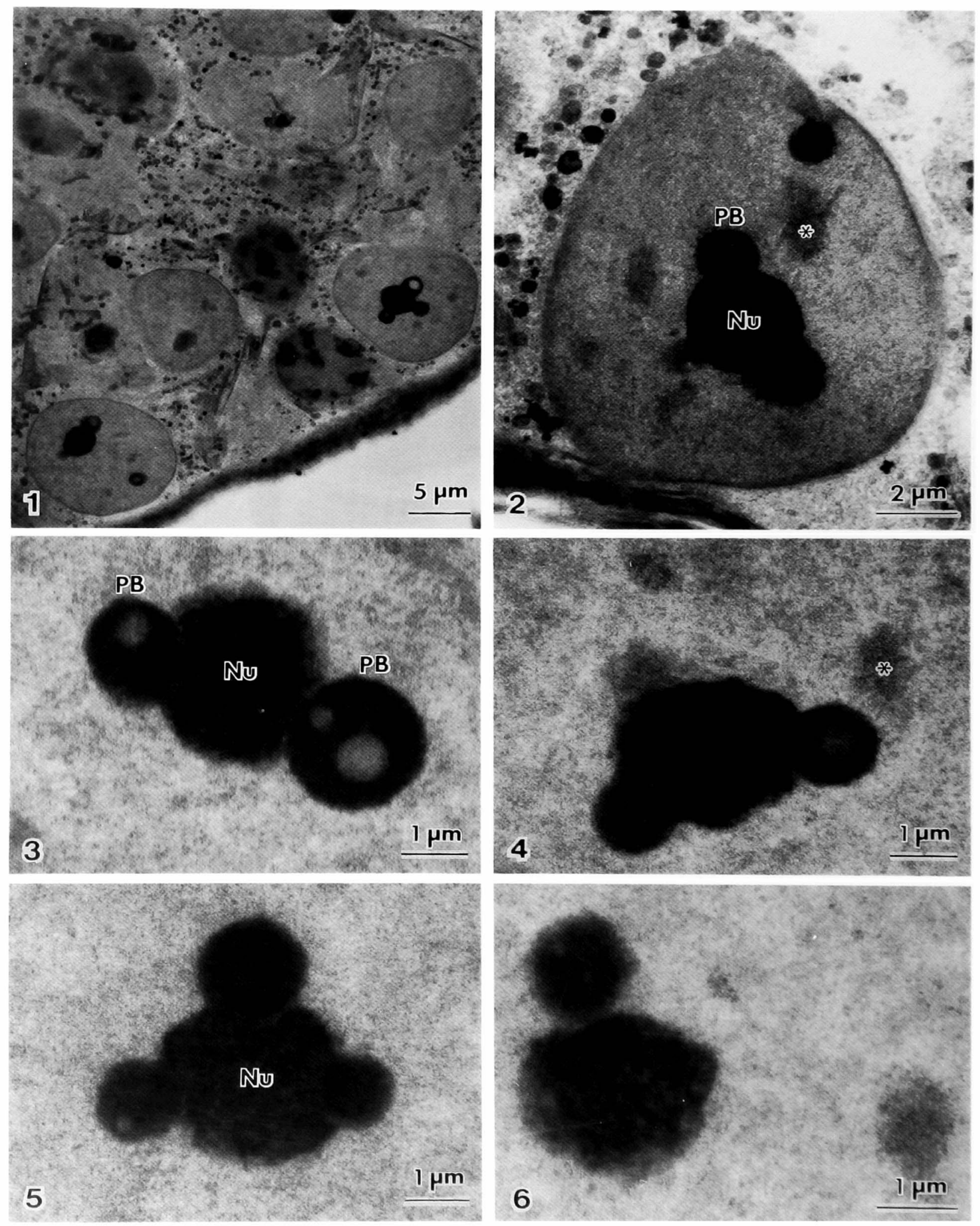


\section{Plate II}

Fig. 7. Electron micrograph of a nucleolar complex, formed by a nucleolus and one perinucleolar body. A $2.0 \mu \mathrm{m}$ section shows two round dense bodies and a small elongated component are seen near the nucleolar complex.

Fig. 8. Electron micrograph of a nucleolar complex without any connection of perinucleolar bodies. Some dense components, including roughly ovoidal, spherical and elongated ones, are shown. $2.0 \mu \mathrm{m}$ section.

Figs. 9 and 10. A stereo pair of a $2.0 \mu \mathrm{m}$ section of nucleolar complex with three perinucleolar bodies associated with the nucleolus. Their complexity is clearly seen. The tilt angle is $\pm 10^{\circ}$.

Figs. 11 and 12. Higher magnification of a $3.0 \mu \mathrm{m}$ section of the Sertoli cell nucleus. Contact between fine fibrillar structures and the nuclcolus is shown (Fig. 11). Fig. 12 also exhibits same structures in the nucleoplasm. Occasionally, it contains small granules. 

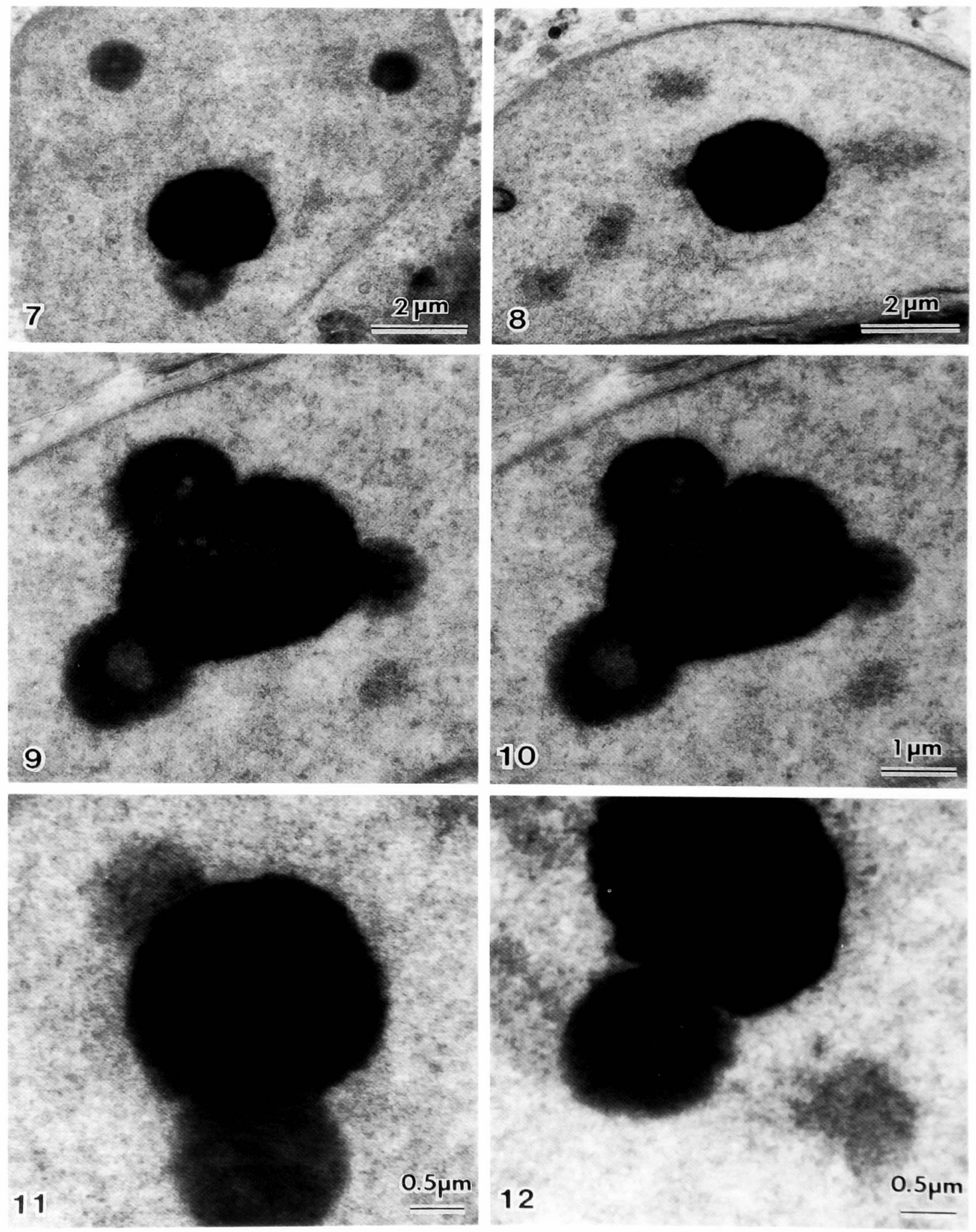